\title{
Unveiling the high-frequency radio source population with the AT20G survey
}

\author{
Elizabeth K. Mahony ${ }^{1}$ on behalf of the AT20G team \\ ${ }^{1}$ ASTRON, the Netherlands Institute for Radio Astronomy, \\ Postbus 2, 7990 AA, Dwingeloo, The Netherlands. \\ email: mahony@astron.nl
}

\begin{abstract}
Until recently, the radio sky above $5 \mathrm{GHz}$ was relatively unexplored. This has changed with the completion of the Australia Telescope $20 \mathrm{GHz}$ survey (AT20G; Murphy et al., 2010); a blind survey of the southern sky down to a limiting flux density of 40 mJy. The AT20G survey provides by far the largest and most complete sample of high-frequency radio sources yet obtained, offering new insights into the nature of the high-frequency active galaxy population. Whilst the radio data provides a unique sample of objects, these data alone are insufficient to completely constrain models of radio source properties and the evolution of radio galaxies. Complementary multiwavelength data is vital in understanding the physical properties of the central black hole.

In this talk I will provide a brief overview of the AT20G survey, followed by a discussion of the multiwavelength properties of the high-frequency source population. In particular, I will focus on the optical properties of AT20G sources, which are very different to those of a low-frequency selected sample, along with the gamma-ray properties where we find a correlation between high-frequency radio flux density and gamma-ray flux density. By studying the multiwavelength properties of a large sample of high-frequency radio sources we gain a unique perspective on the inner dynamics of some of the most active AGN.
\end{abstract}

Keywords. surveys - galaxies: active - radio continuum: galaxies

\section{The AT20G Survey}

The Australia Telescope $20 \mathrm{GHz}$ (AT20G) survey was carried out on the Australia Telescope Compact Array (ATCA) from 2004-2008. The survey was carried out in two phases; a blind survey of the entire southern sky, and a targeted, follow-up survey to obtain accurate positions and flux density measurements. Near-simultaneous observations were also carried out at 5 and $8 \mathrm{GHz}$ to provide reliable spectral index information. For more details on the AT20G survey see Murphy et al. 2010, Massardi et al. 2011 and Hancock et al. 2011.

The final AT20G catalogue consists of 5890 sources above a flux density limit of $S_{20}=$ $40 \mathrm{mJy}$ and is comprised of predominately radio-loud AGN (Murphy et al. 2010, Massardi et al. 2011). Selecting sources at high radio frequencies preferentially selects objects whose emission comes from the central core of the AGN, thus providing insight into the most recent activity. As such, the sample is dominated by compact, flat-spectrum sources.

\section{Optical properties of AT20G sources}

AT20G positions are accurate to 1 arcsec, ideal for multiwavelength crossmatching. Optical identifications of AT20G sources were made using the SuperCOSMOS Science Archive (Hambly et al. 2001) for sources with $|b|>10^{\circ}$. Of these, $78.5 \%$ have optical counterparts, much higher than that seen at lower radio frequencies (typically $25-30 \%$, 
Bock, Large \& Sadler, 1999), and increases with increasing $20 \mathrm{GHz}$ flux density. The detection rate of sources with 'stellar' optical identifications (classified by SuperCOSMOS based on optical morphology) increases as a function of $20 \mathrm{GHz}$ flux density, whereas for sources with 'galaxy' optical counterparts the detection rate decreases with increasing flux density. This suggests that if we were to probe deeper at $20 \mathrm{GHz}$ the galaxy population would become dominant.

Redshift information was found from a range of sources, primarily from the 6dF Galaxy Survey (Jones et al. 2004) or from the literature using the NASA Extragalactic Database (NED). Additional targetted observations were carried out using a range of facilities including Gemini-South, the ESO New Technology Telescope (NTT) and the ANU 2.3m telescope at Siding Spring Observatory. These additional observations resulted in 144 new redshifts. The redshift completeness is quite high for the bright AT20G sources (91.5\% complete for sources brighter than $1 \mathrm{Jy}$ and $85.3 \%$ for sources above $500 \mathrm{mJy}$ ), but this drops off rapidly towards fainter sources. A more detailed description on how optical counterparts and corresponding redshifts were found is presented in Mahony et al. 2011.

Sources where the optical spectrum was available (i.e. sources observed as part of the 6dF Galaxy Survey or from additional observations) formed the spectroscopic sample. This sample was used to study the emission or absorption line properties of AT20G sources. The spectroscopic sample was separated into two broad categories based on their accretion mode. 'Cold-mode' accreting sources (also refered to as High Excitiation Radio Galaxies (HERGs)) are identified by the presence of strong emission features (either narrow or broad), indicating the existance of an efficient, thin accretion disc, dusty torus and narrow and broad line regions; the canonical AGN picture. 'Hot-mode' accretion, or Low Excitiation Radio Galaxies (LERGs), are characterised by weak or no emission lines in the optical spectrum and though to indicate an inefficent mode of accretion which displays no indication of AGN activity in the optical regime (Best \& Heckman, 2012, Hardcastle, Evans \& Croston, 2007).

HERGs exhibit a steeper median radio spectral index than the LERGs. Assuming that the radio emission observed is the combination of the central flat-spectrum core and the steep-spectrum radio jets and lobes, this suggests that the radio jets of these sources dominate the emission, consistent with the idea that these sources are more efficient accretors. This is also shown in Figure 1 which shows that the HERGs have a higher fraction of extended, steep-spectrum sources compared to the LERGs. In addition, for objects with the same absolute magnitude, HERGs have higher radio luminosities than their LERG counterparts, providing further evidence that the 'cold'-mode accreting sources are capable of producing more luminous radio jets and lobes.

\section{Gamma-ray and X-ray properties of AT20G sources}

The gamma-ray properties of AT20G sources were studied using the First Fermi Large Area Telescope (LAT) source catalogue (1FGL; Abdo et al. 2010a). Like the highfrequency radio sky, the gamma-ray sky is dominated by flat spectrum radio sources such as quasars or BL-Lac objects. Crossmatching the 1FGL catalogue with the AT20G catalogue resulted in 233 (43\% of southern Fermi sources) associations within the $95 \%$ Fermi confidence ellipse. The gamma-ray detection rate of AT20G sources increases strongly with $20 \mathrm{GHz}$ flux density, ranging from approximately $1 \%$ at $100 \mathrm{mJy}$ to almost $100 \%$ for flux densities above $10 \mathrm{Jy}$ (Mahony et al. 2010).

The AT20G-1FGL associations agree well with the lower frequency selected AGN catalogue used by the Fermi team to identify gamma-ray counterparts; the First LAT AGN catalogue (1LAC; Abdo et al. 2010b). Eight new Fermi identifications were found 

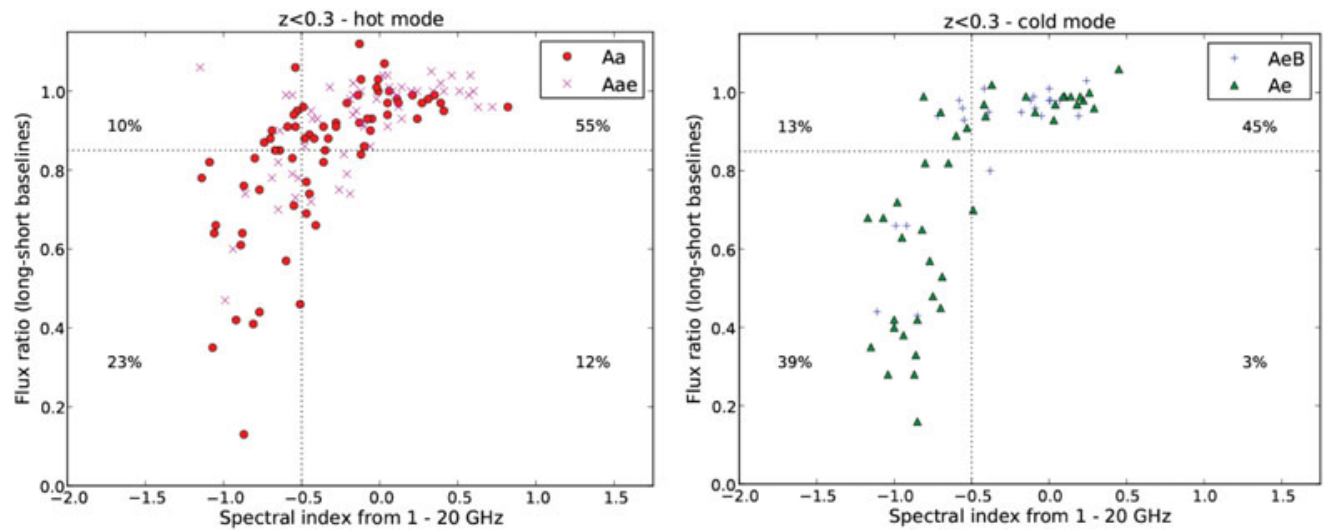

Figure 1. The AT20G sample can be divided into four categories based on the combination of radio spectral index and source size information. The source size is characterised by measuring the ratio of flux densities measured on the long to short ATCA baselines. This allows us to classify sources as being either compact or extended on scales of $\sim 0.15$ arcsec. These figures show the radio spectral indices against the flux ratios for hot-mode accreting sources (LERGS; left) and cold-mode accreting sources (HERGs; right). The vertical line denotes the separation between flat (to the right) and steep (to the left) spectrum sources and the horizontal line marks the separation between compact (above) and extended (below) sources. Only sources with $z<0.3$ are plotted to limit any redshift effects. There is a higher percentage of cold-mode sources in the extended, steep-spectrum quadrant compared to the hot-mode sources. Figure from Mahony et al. 2011.

using AT20G, all at low Galactic latitudes where 1LAC is known to be incomplete. As the AT20G survey provides an unbiased sample with no spectral pre-selection, the agreement with the 1LAC catalogue verifies that there is no missing gamma-ray population of steep spectrum radio sources. A large fraction (87\%) of AT20G sources detected in gammarays have optical identifications, the majority of which are classified as quasars or BLLac objects. A correlation is found between the gamma-ray flux density and $20 \mathrm{GHz}$ flux density. Of the objects classified as variable in the 1FGL catalogue, $87 \%$ are detected in AT20G, much higher than the detection rate of $32 \%$ for the non-variable sources.

X-ray counterparts were identified by crossmatching the AT20G catalogue with the ROSAT All-Sky Survey (RASS; Voges et al. 1999), a survey of the whole sky in the soft $\mathrm{X}$-ray regime $(0.1-2.4 \mathrm{keV})$. This process resulted in 280 matches, corresponding to $3 \%$ of RBSC that have a match in AT20G or $4.7 \%$ of AT20G sources that have an X-ray counterpart. The number of AT20G sources detected in X-rays is similar to the number detected in gamma-rays, however, the overlap in these two samples is quite small; only 56 (1\%) AT20G sources are detected in both X-rays and gamma-rays, a similar fraction to what would be expected by chance. The difference in these populations is illustrated in Figure 2 which plots the spectral index distribution of the full AT20G sample (top panel) compared to the subset of sources with gamma-ray detections (middle panel) and X-ray detections (bottom panel). Kolmogorov-Smirnov tests confirm that these distributions are not drawn from the same parent population at the $99 \%$ confidence level.

\section{Conclusions}

In conclusion, the AT20G survey provides an unprecedented view of the high-frequency radio source population. At these radio frequencies the sky is dominated by flat-spectrum cores of AGN, very different to what is seen at lower radio frequencies. We find a high 


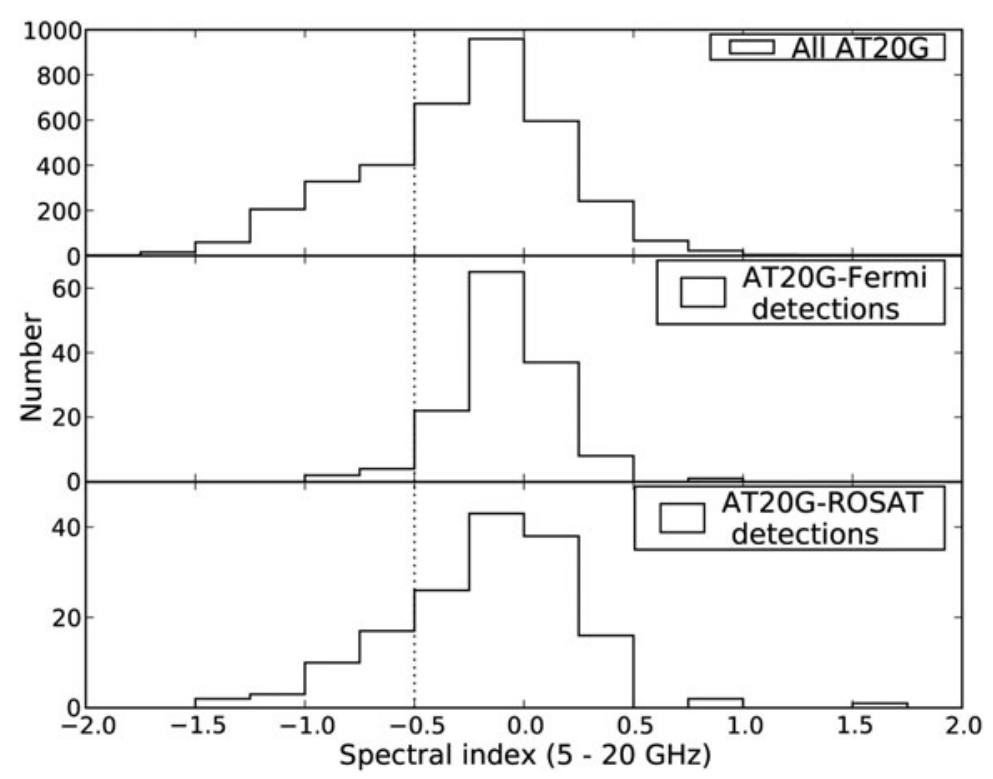

Figure 2. Spectral index distributions for the full AT20G survey (top panel), the subset of AT20G sources detected in gamma-rays by the Fermi mission (middle panel) and the subset of sources detected in X-rays by ROSAT (bottom panel). Figure from Massardi et al., 2011.

optical identification rate of $78.5 \%$ (for optical magnitudes brighter than $\mathrm{B}=22$ ) and see evidence that more efficiently accreting sources can produce stronger radio jets. A high fraction of southern Fermi gamma-ray sources are detected in AT20G and we find a strong correlation between the radio and gamma-ray flux densities. A similar number of X-ray matches are found, but these form a different population to the gamma-ray counterparts.

\section{References}

Murphy, T., et al. 2010, MNRAS, 402, 2403

Massardi, M., et al. 2011, MNRAS, 412, 318

Hancock, P. J., et al. 2011, ExA, 32, 147

Hambly, N. C., et al. 2001, MNRAS, 326, 1279

Bock, D., Large, M. I., \& Sadler, E. M. 1999, AJ, 117, 1578

Jones, D. H., et al. 2004, MNRAS, 355, 747

Mahony, E. K., et al. 2011, MNRAS, 417, 2651

Best, P. N. \& Heckman, T. M. 2012, MNRAS, 421, 1569

Hardcastle, M. J., Evans, D. A., \& Croston, J. H. 2007, MNRAS, 376, 1849

Abdo, A. A., et al. 2010a, ApJS, 188, 405

Mahony, E. K., et al. 2010, ApJ, 718, 587

Abdo, A. A., et al. 2010b, ApJ, 715, 429

Voges, W., et al. 2010, A\&A A, 718, 587 